\title{
Online Games to Improve Children's Knowledge of Nutrition and Physical Activity Guidelines - A Pilot Study
}

\author{
Natalie Rangelov ${ }^{1}$, L. Suzanne Suggs ${ }^{1}$, Aurora Occa ${ }^{1}$, Olga Radchuk ${ }^{1} \&$ Andreas Schmeil ${ }^{1}$ \\ ${ }^{1}$ BeCHANGE Research Group, Institute for Public Communication, Faculty of Communication Sciences, \\ Università della Svizzera italiana, Lugano Switzerland \\ Correspondence: Natalie Rangelov, BeCHANGE Research Group, Institute for Public Communication, Università \\ della Svizzera italiana - Via G. Buffi 13, CH - 6900 Lugano, Switzerland. E-mail: natalie.rangelov@gmail.com
}

Received: January 2, 2017 Accepted: February 14, 2017 Online Published: February 28, 2017

doi:10.5539/gjhs.v9n4p134 URL: https://doi.org/10.5539/gjhs.v9n4p134

\begin{abstract}
The benefits of a healthy lifestyle during childhood are difficult to overestimate and computer games represent a promising intervention to promote healthy lifestyles in children. The purpose of this pilot study was to examine the effects of online games for improving children's knowledge of healthy nutrition and physical activity (PA) guidelines. Using an experimental design, we aimed to understand if children aged 7-9 liked this type of intervention, if baseline knowledge of PA or nutrition guidelines improved after playing one of two games, and beyond those of the control condition, and if one game was more effective than the other. Each intervention, including the control, resulted in positive change, showing an overall improved knowledge. The game with the greatest improvements was the more interactive game that gave feedback along the way. Our pilot study showed that games may be useful in promoting healthy lifestyles to children.
\end{abstract}

Keywords: games, children, child-computer interaction, lifestyle behavior, nutrition, physical activity

\section{Introduction}

Unhealthy diet and insufficient physical activity are linked to preventable health related problems, including overweight and obesity among many others. Health behaviors in childhood are associated with health behaviors in adulthood and food preferences of children form the basis for their future eating behavior (Cooke, 2007). The benefits of a healthy lifestyle during childhood are difficult to overestimate and make interventions aimed at improving knowledge of healthy behaviors especially important (Anderson, Baird, Davis Jr, Ferreri, Knudtson, Koraym, Waters, \& Williams, 2009).

Computer games represent an opportune media-based intervention to promote healthy lifestyles (Baranowski, Buday, Thompson, \& Baranowski, 2008). Educational games allow children to gain knowledge while having fun. Studies have shown positive effects of entertainment-education health programs and their ability to improve knowledge, enhance existing skills, develop new skills, and change behavior (Baranowski et al., 2008).

Computer games can differ in format and design, and vary in level of interactivity. Interactivity refers to "the degree to which users of a medium can influence the form or content of the mediated environment" (Steuer, 1995). Previous research has shown that interactivity is associated with educational and learning outcomes. According to Ritterfeld et al. (Ritterfeld, Shen, Wang, Nocera, \& Wong, 2009), players' participation and engagement can be enhanced, both from a behavioral aspect, and from a cognitive one. Through interactivity, learning interest can be stimulated, and give the possibility for a more active leaning process (Kennedy \& Kennedy, 2004; Ritterfeld et al., 2009; Schneiter \& Gerjets, 2007; Wouters, Tabbers, \& Paas, 2007). Thus using computer games to teach children about healthy nutrition and physical activity is a promising approach as these games may be used to convey important messages about physical activity and healthy eating in an unobtrusive context (Lin, Mamykina, Lindtner, Delajoux, \& Strub, 2006; Thompson, Baranowski, Buday, Baranowski, Thompson, Jago, \& Griffith, 2010).

The purpose of this pilot study was to examine two online games for improving children's knowledge of the Swiss guidelines for healthy nutrition and physical activity (PA) behaviors. We aimed to understand if children liked this type of intervention, if baseline knowledge of PA or nutrition guidelines improved after playing one of two games, and beyond those of the control condition, and if one game was more effective than the other one, given the difference in design between the two games. The specific research questions are: 
1) To what extent did children like these interventions?

2) To what extent did baseline knowledge of PA and nutrition guidelines improve after the intervention, for each of the study groups?

3) To what extent did the effects differ for each study group?

This is the first published study reporting the effects of online games for healthy nutrition and physical activity guidelines knowledge attainment of Swiss children.

\section{Methods}

\subsection{Study Design}

An experimental design was employed to test the effects of two online games on knowledge improvement of guidelines for nutrition and PA for children ages 7-9. This age range was chosen for two reasons. First, the Swiss Society for Nutrition (SSN) guidelines for healthy nutrition for children are divided by age groups: 6 years of age, 7-9 years of age, and 10-12 years of age (Swiss Society for Nutrition, 2010). Second, the existing games were best suited for the 7-9 year old cohort. The existing games we used to base our intervention on required reading skills and pre-tests suggested they were "too young" for 10-12 year old audiences. Children of 7-9 years of age were able to read and play the games autonomously.

The two tested games offered the same content related to nutrition and PA guidelines, but in a different format (see below section 2.5 for details about the two games). We hypothesized that playing the online health game "The Millionaire" would lead to higher increase of knowledge about PA and nutrition guidelines when compared to the online game "Fill-the-Gaps" or the non-interactive text used for the control group. Participant children aged 7-9 were randomly assigned to control group or one of two game groups.

\subsection{Participants and Recruitment}

To participate in this pilot study, children must have been residing in the Canton Ticino (Switzerland's most southern state) and must have been willing to participate in a study session at the University. According to Swiss Law, ethics approval was not required for this study. However, in accordance with the Helsinki Declaration, parental and child consent were both obtained before data collection began.

For this pilot study, a purposive non-probability sampling technique was used. Participants were recruited from a list of families who participated in a previous study conducted by the authors (Rangelov \& Suggs, 2015) and who consented to be invited to participate in future studies. An e-mail was sent to parents on May 2, 2013, inviting them and their children to participate in this study between May 13 - 31,2013. A link to Doodle scheduler was included in the invitation where sessions were scheduled by appointment. In the scheduler application, participant's identity was concealed; timeslots only showed if they were available or booked. For those who did not reply, a second invitation was e-mailed on May 7, 2013 with a final invitation one week later.

\subsection{Procedures}

Upon arrival at the University, participants were welcomed and accompanied to the study room by a research assistant. Both parents and children were provided with an oral explanation about the purpose of the study and the steps needed to complete it. They were told that they were allowed to withdraw from the study at any time, without providing a reason. Parents and children were provided with a written explanation of the study details, and asked to sign a statement of informed consent before starting the session.

During the session, parents and children stayed in the same room, but sat at a distance from each other with the parent being out of direct sight of the child, and wearing headphones. This was done to help children and parents feel comfortable, while the distance and the headphones should have eliminated the influence of parents on their child's answers to the questionnaire and while playing the assigned game. Parents were asked not to interact with the children during the session.

The study condition was randomly assigned by having the child take out a numbered piece of paper from a bag. The numbers ranged from 1 to 3, with 1 being "Control Group" (G1), 2 - the online game "Fill-the-Gaps" (G2) and 3 - the online game "The Millionaire" (G3). The study assistant then asked all children to complete the pre-study questionnaire. The control group participants were asked to read a text reporting the nutrition and physical activity recommendations, and then proceed to the post-study questionnaire. Game groups were introduced to one of the two games followed by the same post-study questionnaire. Once completed the post-study questionnaire, parents and children were offered a piece of fruit, and children were able to take one gift item, choosing from a jump rope, a ball, a hat, a sticker or a key holder. 


\subsection{The Guidelines}

The Swiss Society for Nutrition (SSN) provides recommendations for food and drink consumption behaviors that vary according to age (Swiss Society for Nutrition, 2010). These guidelines were developed by SSN to be used by the lay population, including parents and children. For the purpose of this study, knowledge about nutrition guidelines for six categories was assessed: meat, dairy products, fruit $\&$ vegetables, grains, sweets $\&$ snacks, and water. See Table 1 for these guidelines for children ages 7-9.

Consistent with the World Health Organization, the Swiss Federal Office of Sport (FOSPO) recommends a minimum of 60 minutes of moderate-to-vigorous physical activity daily for children. They also recommend physical activity after every two hours of sedentary behavior (Federal Office of Sport \& Réseau suisse Santé et activité physique (hepa.ch), 2013).

Table 1. Nutrition Guidelines Assessed in This Study

\begin{tabular}{lll}
\hline Food & Portion & Frequency \\
\hline Meat (of which salami max. 1 portion) & 5 portions max. & Weekly \\
Dairy products & $2-3$ portions & Daily \\
Fruit & 2 portions & Daily \\
Vegetables & 3 portions & Daily \\
Grains & $3-4$ portions & Daily \\
Sweets \& snacks & 1 portion max. & Daily \\
Water, fruit or herbal sugar free tea & 9 dl & Daily \\
\hline
\end{tabular}

\subsection{The interventions}

The text for the control group (G1) was a list of sentences reporting the Swiss nutrition and PA recommendations (see Figure 1). To keep the messenger and the delivery method consistent with those in the treatment groups, the text was placed on a webpage and included the messenger "Larry" (bigger character on top left and bottom right, in Figure 1) and the "audience" images (smaller images on top right, in Figure 1) from the online games.

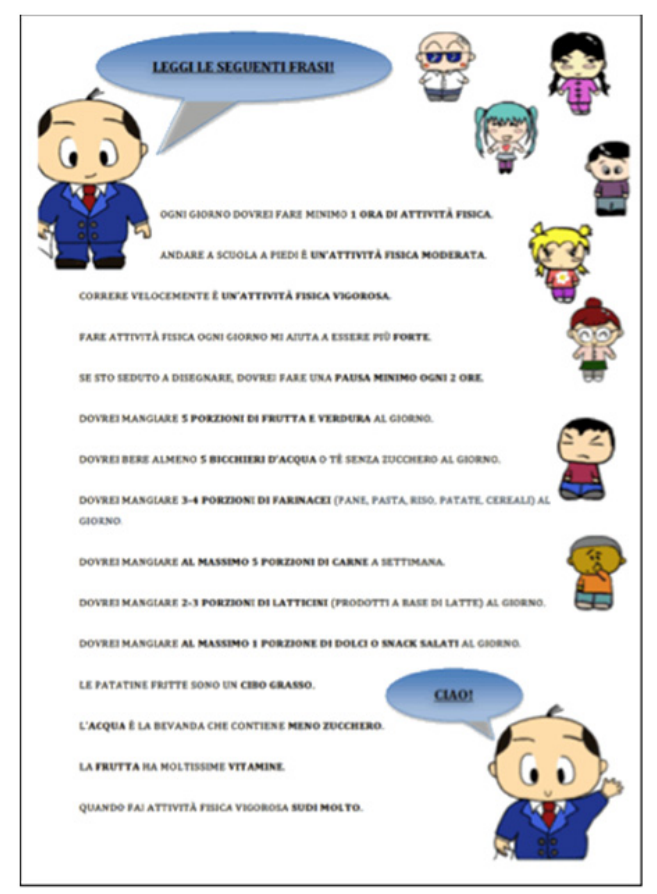

Figure 1. Text for control group 
Similar to "Mad-libs" (Stern, 2014), the online game "Fill-the-Gaps" (G2) required participants to read statements about the Swiss nutrition and PA recommendations with blanks in the sentences and then to fill the blanks. Using the drag-and-drop feature, they had to choose the answer from a list of choices, drag it to the correct place in the text using a computer mouse and fill the empty space (see Figure 2). The text, presented in the game, was the same as in the control group, but missing one or two words in each sentence. The layout presented "Larry", the moderator of the game (on the left, in Figure 2), and the "audience" (under the text, in Figure 2). There was no set time limit and participants could change their answer at any time before completing the game. The player could use a help option: upon clicking the button "Help" ("Aiuto"), the first letter of the unknown word appeared in the gap. This option could be used multiple times. When all the gaps were filled, the player was required to press the "Finish" ("Finito") button. Feedback was provided at the end of the game. Answers placed correctly turned into a plain text. Wrong answers stayed in the form of text boxes. The game provided a score with a remark, stating whether all the answers were placed correctly or if revision was recommended. In the latter case, the player had the opportunity to revise their answers and submit them again.

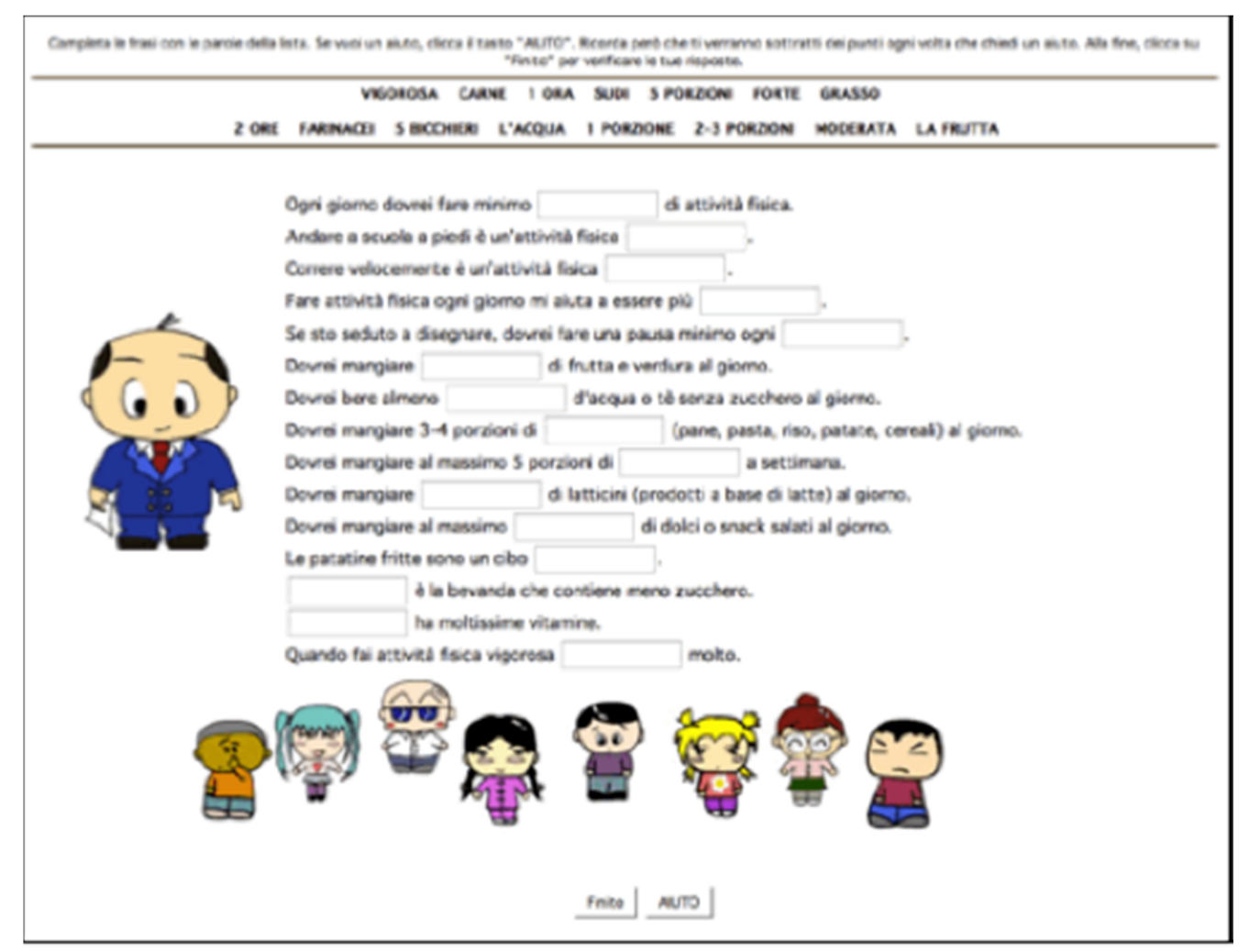

Figure 2. Online Game "Fill-the-Gaps"

The third group played the Millionaire game (G3), inspired by the TV game show "Who wants to be a millionaire?" The interactive on-line game contained the same guidelines, presented one-by-one with a two-minute time frame to respond. Each was in the form of a question and accompanied by four possible answers, only one of which was correct. Each correct answer allowed the player to win hypothetical money. The moderator "Larry" presented the questions and guided the game, and the "audience" reacted to answers. Compared to the Fill-the-Gaps game, feedback on children's answers was given immediately. Each correct answer appeared in a green frame and was accompanied by the moderator's comment "Correct! You're doing very well!" (“Giusto! Sei bravissimo/a!"), along with applause from the audience. Each wrong answer appeared in a red frame and was accompanied by the comment "Unfortunately the answer is not correct" ("Purtroppo sbagliato...") and the audience sighed. When a wrong answer was given or the participant ran out of time to answer a question, the player had to start the game over. The player could use several help options, multiple times: ask the audience for help, exclude two wrong answers, and get additional two minutes of time. 


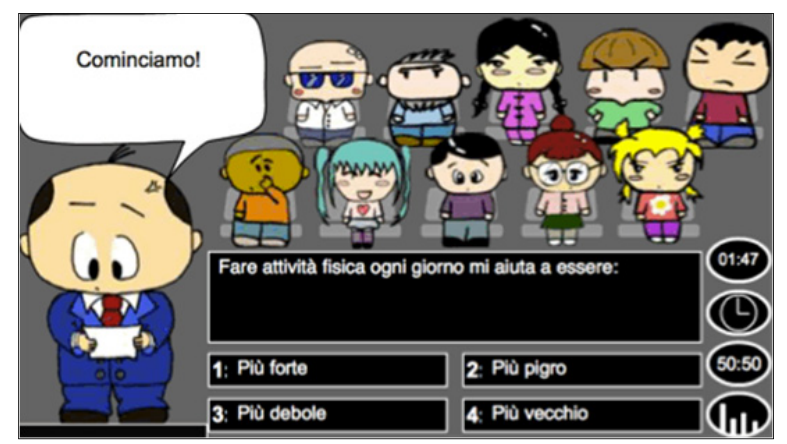

Figure 3. Online Game "The Millionaire"

\subsection{Outcome Measures}

Outcome measures included children's satisfaction with the games and text, as well as knowledge of nutrition and PA guidelines. Satisfaction was measured at post-study questionnaire using three items assessing liking, wanting to play again, and perceived difficulty. Knowledge about nutrition guidelines was assessed at pre and post-test using six items with true/false/I do not know response options: 1) I should eat a maximum of 3 portions of meat per week; 2) I should eat 2-3 portions of dairy products per day; 3 ) I should eat at least 5 portions of fruit and vegetables a day; 4) I should eat 3-4 portions of starchy foods (bread, pasta, rice, potato, cereal) per day; 5) I should drink at least 2 glasses of water or tea without sugar per day; and 6) I should eat a maximum of 1 portion of sweets or salty snacks per day. PA guideline knowledge was assessed at pre and post using two items: 1) I should practice a minimum of 3 hours of physical activity per day; and 2) If I am sitting and drawing, I should have a break minimum every 5 hours, both with true/false/I do not know response options.

\subsection{Data Analysis}

Data were analyzed using SPSS v.21 (SPSS Inc., Chicago, IL, USA). Descriptive statistics show pre and post results in all three groups. Ratio scores for knowledge by group were calculated summing up individual scores of each child in the same intervention group, and dividing it by the number of children in the intervention group, in order to account for the different group sizes. Differences in ratio scores were computed, to determine the extent of the change in knowledge.

\section{Results}

\subsection{Sample Characteristics}

The pilot study sample characteristics are shown in Table 2.

Table 2. Sample Characteristics

\begin{tabular}{lll}
\hline Sample $(\mathrm{N}=19)$ & $\mathrm{n}$ & $\%$ \\
\hline Gender & 11 & 57.9 \\
$\quad$ Male & 8 & 42.1 \\
$\quad$ Female & & \\
Age $($ mean $=8.11$ years; range $=7-9 ; \mathrm{SD}=.875)$ & 6 & 31.6 \\
$\quad 7$ years & 5 & 26.3 \\
8 years & 8 & 42.1 \\
9 years & & \\
Grade at school & 3 & 15.8 \\
$1^{\text {st }}$ grade & 6 & 31.6 \\
$2^{\text {nd }}$ grade & 7 & 36.8 \\
$3^{\text {rd }}$ grade & 3 & 15.8 \\
$4^{\text {th }}$ grade & & \\
\hline
\end{tabular}


Five children (26.3\%) were randomly assigned to the control group (G1), eight (42.1\%) to the "Fill-the-Gaps" game (G2), and six (31.6\%) to "The Millionaire" game (G3). There were three females and two males in G1, five females and three males in G2, and three female and three male participants in G3. The mean age in G1 was 8.00 (range $=7-9$, $\mathrm{SD}=.707$ ); in $\mathrm{G} 2$ it was 8.13 (range $=7-9 ; \mathrm{SD}=.991$ ); and in $\mathrm{G} 3$ the mean age was 8.17 (range 7-9; $\mathrm{SD}=.983$ ).

\subsection{Satisfaction}

Thirteen children out of 19 (68.4\%) answered the questions related to satisfaction. Overall, most children (11 children, 84.6\%) reported having liked the intervention they were assigned to; two children (15.4\%) answered "I do not remember". Most children (11 children, 84.6\%) reported interest in conducting again the experiment (no matter of the groups of assignment); one child said "no" (7.7\%), and one answered "I do not know" (7.7\%). For most children (9 children, $69.2 \%$ ) what they were asked to do (read the text or play one of the two games) was not difficult; for three, it was difficult (23.1\%), and one said s/he did not remember (7.7\%).

Three children in G1, one in G2, and two in G3 did not answer the post-survey. In G1 one child stated liking the text and being interested in reading it again, and one child stated that s/he did not like it and did not want to repeat it. Both children reported the text as easy to read. In G2, six children (85.7\%) stated they liked the "Fill-the-Gaps" game and that they were interested in playing it again; one liked it so-so (14.3\%) and did not want to play it again. Four children (57.1\%) reported that the game was not difficult; two children $(28.6 \%)$ found it difficult to play and one did not remember (14.3\%). In G3, all four children that answered the post-survey reported having liked "The Millionaire" game and being willing to play it again. Three of them (75\%) reported that the game was not difficult, while one (25\%) child said it was difficult to play.

\subsection{Knowledge of Nutrition and Physical Activity Guidelines}

Most children (14/19 children, 73.7\%) improved their score from baseline to post-test experiment. In Table 3, percentages of correct answers and ratio scores at baseline and follow-up are shown, as are differences in percentages pre and post experiments, and differences in ratio scores.

Table 3 .

\begin{tabular}{lllllll}
\hline & Baseline $\%$ & Ratio score & Follow up \% & Ratio score & Difference $\%$ & $\begin{array}{l}\text { Difference } \\
\text { ratio }\end{array}$ \\
\hline G1 & $42.5 \%$ & 3.40 & $57.5 \%$ & 4.60 & $15 \%$ & +1.20 \\
G2 & $42.2 \%$ & 3.38 & $67.2 \%$ & 5.38 & $25 \%$ & +2.00 \\
G3 & $41.7 \%$ & 3.33 & $72.9 \%$ & 5.83 & $31.25 \%$ & +2.50 \\
\hline
\end{tabular}

Considering the difference in skills according to the grade attended at school, the results are the following: children in $1^{\text {st }}$ grade showed an improvement with a ratio of 1.67 , and children in $2^{\text {nd }}$ grade showed an improvement with a ratio equal to 0.83 . The children in $3^{\text {rd }}$ grade showed an improvement with a ratio of 3.00 , and children in fourth grade showed an improvement with a ratio of 2.00 .

\section{Discussion}

This pilot study examined two online games as tools to improve knowledge of the recommendations for health enhancing nutrition and physical activity behaviors for children aged 7 to 9 years in southern Switzerland. The baseline knowledge for both nutrition and physical activity guidelines was similar for all three groups of children (G1, G2, and G3). Each intervention, including the control, resulted in positive change, showing an overall improved knowledge of Swiss nutrition and physical activity recommendations.

The groups with the greatest improvements were those of the Fill-in-the-gaps game and of the Millionaire game, with a slightly higher improvement for the children assigned to this last, more interactive game. This is consistent with previous studies, suggesting that educational games, and in particular interactivity and participants' participation and engagement are associated with educational attainment (Baranowski et al., 2008; Ritterfeld et al., 2009). Related to satisfaction, regardless of the experiment they were assigned to, most children liked the intervention and thought it was not difficult. Most of them were also willing to repeat it. Thus, this pilot study showed that the games employed served as valuable tools for communicating health with children, a finding consistent with previous studies (Lin et al., 2006; Thompson et al., 2010). However, further research is needed. Indeed, our findings suggest that all games are helpful for this age group, and we are unable to conclude that one game was more effective than the other, or if effects differ by subgroups of children. 


\subsection{Limitations of the Study}

This pilot study should be interpreted in light of its limitations. It followed an experimental study design, in which small samples are common, but it is not generalizable without further examination and larger samples. The small sample size did not assure equal distribution of gender and age (or grade) of children. Because of the small sample, more advanced analyses were not appropriate; hence, the statistical power and significance were not calculated.

Regarding the design of the experiments, the three games presented several differences in format and function, hence it is difficult to state which characteristics of the game were associated with learning. Also, the games were played only once, while typical games are designed to be played repeatedly and many build on previous user experiences with the game. Additionally, while children in the three groups were equally distributed by age, the control condition game was text only and thus the results obtained may have been influenced by the reading skills of the children. Finally, the setting of the experiment was a university room, which is quite "artificial", compared to the usual setting where children live and play (i.e. home, school, etc.).

\section{Conclusion}

Our pilot study showed that using games as a strategy for improving knowledge of health behavior guidelines in Italian speaking Swiss children ages 7-9 may be useful, but that the way forward is not evident. Before determining if one communication strategy is worth greater investment, this study needs to be replicated with a larger, representative sample of children. We also need opportunities for children to play the game multiple times over a period of time, and in real life settings (i.e., home) rather than a university meeting room. Longer-term assessment of outcomes, including behavior change must be included in this next step.

While the names of games, the channels and technologies games are played on, and how games are played varies across cultures and countries, children's interest in playing games is universal. The games that were used in this study are easily adaptable to other countries and channels (e.g., mobile phones), and nutrition and physical activity recommendations. Thus, further research might compare the learning and behavioral outcomes of similar experiments with children from different countries. Learning outcomes could be influenced by culture, school systems and policies related to children's health education. This would result in understanding to what extent are these games worth investment and for what target audiences.

Despite the study limitations, we have learned that games and child-friendly text can be helpful in improving knowledge of health behavior recommendations for children. However, single exposure to any information is not likely to result in sustained behavior change. Games have such potential, if they are fun and engaging (Baranowski et al., 2008; Lin et al., 2006; Thompson et al., 2010). Games are not likely to be the sole solution to poor childhood nutrition and physical activity behaviors, but they may be a beneficial part of the puzzle.

\section{Acknowledgments}

We sincerely thank the participants in this study and the parents who consented to their children taking part. We also thank our colleagues in the e-Lab at the Università della Svizzera italiana (USI) who originally developed the games used in our study. We modified the games for health recommendations in Switzerland.

\section{Author Disclosure}

We have no conflict of interest to declare. The study was part of a master thesis study of OR and all authors participated in that study design, study conduct and the writing of this paper. All authors agree on the order of authors.

\section{References}

Anderson, J. W., Baird, P., Davis Jr, R. H., Ferreri, S., Knudtson, M., Koraym, A., Waters, V., \& Williams, C. L. (2009). Health benefits of dietary fiber. Nutrition Reviews, 67(4), 188-205. https://doi.org/10.1111/j.1753-4887.2009.00189.x

Baranowski, T., Buday, R., Thompson, D. I., \& Baranowski, J. (2008). Playing for Real: Video Games and Stories for Health-Related Behavior Change. American Journal of Preventive Medicine, 34(1), 74-82.e10. https://doi.org/10.1016/j.amepre.2007.09.027

Cooke, L. (2007). The importance of exposure for healthy eating in childhood: A review. Journal of Human Nutrition and Dietetics, 20(4), 294-301. https://doi.org/10.1111/j.1365-277X.2007.00804.x

Federal Office of Sport, \& Réseau suisse Santé et activité physique (hepa.ch). (2013, November 15). Recommandations d'activité physique. Retrieved March 4, 2014, from http://www.hepa.ch/internet/hepa/it/home/dokumentation/dokumente_bewegung.parsys.87397.downloadLis 
t.17891.DownloadFile.tmp/bewegungsempfehlungenkinderi.pdf

Kennedy, G., \& Kennedy, G. (2004). Promoting Cognition in Multimedia Interactivity Research. Journal of Interactive Learning Research, 15(1), 43-61.

Lin, J. J., Mamykina, L., Lindtner, S., Delajoux, G., \& Strub, H. B. (2006). Fish'n'Steps: Encouraging Physical Activity with an Interactive Computer Game. In P. Dourish \& A. Friday (Eds.), UbiComp 2006: Ubiquitous Computing (pp. 261-278). Springer Berlin Heidelberg. Retrieved from http://link.springer.com/chapter/10.1007/11853565_16, https://doi.org/10.1007/11853565_16

Rangelov, N., \& Suggs, L. S. (2015). Using Strategic Social Marketing to Promote Healthy Nutrition and Physical Activity Behaviors to Parents and Children in Switzerland: The Development of FAN. Cases in Public Health Communication \& Marketing, 8, 27-50.

Ritterfeld, U., Shen, C., Wang, H., Nocera, L., \& Wong, W. L. (2009). Multimodality and Interactivity: Connecting Properties of Serious Games with Educational Outcomes. Cyber Psychology \& Behavior, 12(6), 691-697. https://doi.org/10.1089/cpb.2009.0099

Schneiter, K., \& Gerjets, P. (2007). Learner Control in Hypermedia Environments. Educational Psychilogy Review, 19(3), 285-307. https://doi.org/10.1007/s10648-007-9046-3

Stern, L. (2014). Mad Libs. Retrieved June 23, 2014, from http://www.madlibs.com/

Steuer, J. (1995). Defining Virtual Reality: Dimensions Determining Telepresence. In Communication in the Age of Virtual Reality (Edited by Biocca F., and Levy M.R.). Routledge.

Swiss Society for Nutrition. (2010). Società Svizzera di Nutrizione: L'alimentazione dei bambini. Retrieved April 20, 2011, from http://www.sge-ssn.ch/it/io-e-te/mangiare-e-bere/giovani-e-anziani/bambini/

Thompson, D., Baranowski, T., Buday, R., Baranowski, J., Thompson, V., Jago, R., \& Griffith, M. J. (2010). Serious Video Games for Health: How Behavioral Science Guided the Development of a Serious Video Game. Simulation \& Gaming, 41(4), 587-606. https://doi.org/10.1177/1046878108328087

Wouters, P., Tabbers, H. K., \& Paas, F. (2007). Interactivity in Video-based Models. Educational Psychology Review, 19(3), 327-342. https://doi.org/10.1007/s10648-007-9045-4

\section{Copyrights}

Copyright for this article is retained by the author(s), with first publication rights granted to the journal.

This is an open-access article distributed under the terms and conditions of the Creative Commons Attribution license (http://creativecommons.org/licenses/by/4.0/). 